\title{
Initial experience with AcQMap catheter for treatment of persistent atrial fibrillation and atypical atrial flutter
}

\author{
M. Liebregts (D) - M. C. E. F. Wijffels · M. N. Klaver · V. F. van Dijk • J. C. Balt • L. V. A. Boersma
}

Accepted: 16 August 2021 / Published online: 26 October 2021

(C) The Author(s) 2021

\begin{abstract}
Introduction The AcQMap High Resolution Imaging and Mapping System was recently introduced. This system provides 3D maps of electrical activation across an ultrasound-acquired atrial surface.

Methods We evaluated the feasibility and the acute and short-term efficacy and safety of this novel system for ablation of persistent atrial fibrillation (AF) and atypical atrial flutter.

Results A total of 21 consecutive patients (age (mean \pm standard deviation) $62 \pm 8$ years, 23\% female) underwent catheter ablation with the use of the AcQMap System. Fourteen patients $(67 \%)$ were treated for persistent $\mathrm{AF}$ and 7 patients (33\%) for atypical atrial flutter. Eighteen patients (86\%) had undergone at least one prior ablation procedure. Acute success, defined as sinus rhythm without the ability to provoke the clinical arrhythmia, was achieved in 17 patients $(81 \%)$. At 12 months, 4 patients treated for persistent AF (29\%) and 4 patients treated for atypical flutter $(57 \%)$ remained in sinus rhythm. Complications included hemiparesis, for which intra-arterial thrombolysis was given with subsequent good clinical outcome $(n=1)$, and complete atrioventricular block, for which a permanent pacemaker was implanted
\end{abstract}

\footnotetext{
Supplementary Information The online version of this article (https://doi.org/10.1007/s12471-021-01636-w) contains supplementary material, which is available to authorized users.

M. Liebregts $(\bowtie) \cdot$ M. C. E. F. Wijffels · M. N. Klaver

V. F. van Dijk · J. C. Balt · L. V. A. Boersma

Department of Cardiology, St. Antonius Hospital,

Nieuwegein, The Netherlands

maxliebregts@gmail.com

\section{V. A. Boersma}

Department of Cardiology, Amsterdam University Medical

Centers, Amsterdam, The Netherlands
}

$(n=2)$. No major complications attributable to the mapping system occurred.

Conclusion The AcQMap System is able to provide fast, high-resolution activation maps of persistent AF and atypical atrial flutter. Despite a high acute success rate, the recurrence rate of persistent $\mathrm{AF}$ was relatively high. This may be due to the selection of the patients with therapy-resistant arrhythmias and limited experience in the optimal use of this mapping system that is still under development.

Keywords Atrial fibrillation · Atrial flutter · Mapping system · Catheter ablation

\section{Introduction}

Pulmonary vein isolation (PVI) for the treatment of paroxysmal atrial fibrillation (AF) and cavotricuspid isthmus ablation for the treatment of typical atrial flutter are straightforward procedures with high success rates [1, 2]. Additional non-PV triggers respon-

\section{What's new?}

- This is the first study describing real-world outcomes in patients treated for persistent atrial fibrillation (AF) and atypical atrial flutter with the use of the AcQMap system.

- The AcQMap system provided a high acute success rate $(81 \%)$.

- No recurrence of clinical arrhythmia was observed in $86 \%$ of the patients treated for atypical atrial flutter during a follow-up of 12 months.

- Only $29 \%$ of the patients treated for persistent AF remained in sinus rhythm after a follow-up of 12 months. 
sible for driving and maintaining $\mathrm{AF}$ are thought to exist in persistent AF [3-5]. This explains why PVI alone renders a much lower success rate in these patients $[6,7]$. Current point-by-point mapping systems are unable to accurately display complex arrhythmias because of the sequential nature by which such systems acquire, post-process and display voltage-based contact signals. Therefore, they are not well suited to locate and eliminate the drivers and maintenance mechanisms of persistent AF.

We report our first experience with a novel noncontact ultrasound array catheter for mapping and treating persistent $\mathrm{AF}$ and atypical atrial flutter.

\section{Methods}

\section{Patient selection and study design}

All patients who were treated for persistent AF or atypical atrial flutter with the use of the AcQMap High Resolution Imaging and Mapping System (Acutus Medical, Carlsbad, CA, USA) at the St. Antonius Hospital Nieuwegein, the Netherlands between December 2016 and December 2018 were included. The procedures were conducted by one or two operators (MCEFW and/or LVAB). All patients had their first clinical check-up at 3 months and were followed up for a maximum of 12 months. Patients who underwent a redo procedure were excluded from further follow-up. Baseline characteristics, procedural data and outcomes were retrospectively collected from hospital patient records.

\section{Description of the system}

The AcQMap System has the ability to rapidly generate $3 \mathrm{D}$ ultrasound maps of heart chambers overlaid with high-resolution activation maps. The electrical activation can be shown as voltage or dipole density. Voltage maps are made up of the summation of local charges arising from cardiac cells all over the myocardium. Dipole density maps (Coulombs $/ \mathrm{cm}^{2}$ ) represent the magnitude of these sources upon the endocardial surface and are therefore more precise [8]. The AcQMap System acquires dipole density from noncontact sensing of cardiac voltage within the heart chamber as a whole. This results in a global view of the conduction pattern of every activation cycle and permits the acquisition of consecutive activation maps on a beat-to-beat basis [9].

The diagnostic recording catheter (AcQMap 3D Imaging and Mapping Catheter) consists of six splines, which are populated with eight ultrasound transducers and eight biopotential electrodes each, making up a total of 48 sensors of each type. The catheter is deployed by the user into the left or right atrium as a spheroidal basket (Fig. 1). Patch electrodes are attached to the patient to provide localisation data to the system. To create an anatomical map, the user

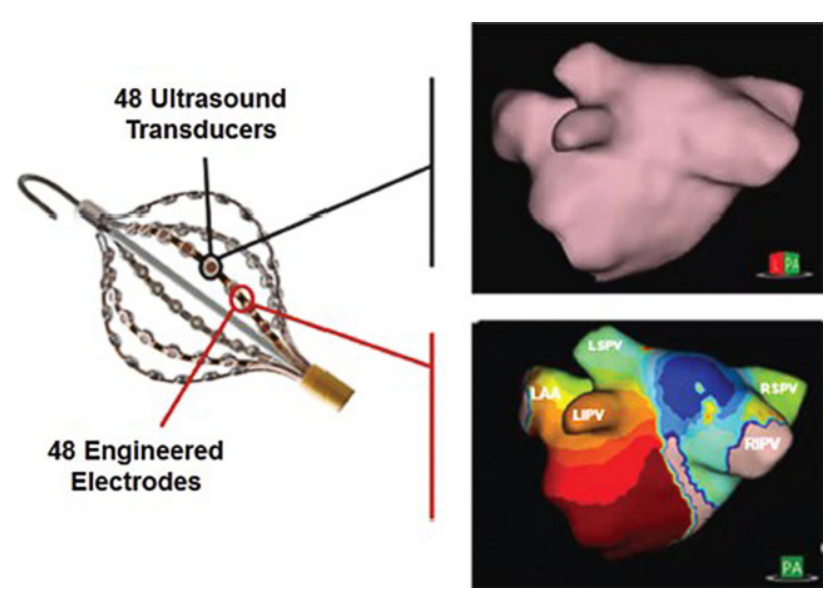

Fig. 1 AcQMap catheter. The six splines of the catheter have 48 ultrasound transducers for anatomy reconstruction (upper right panel) and 48 electrodes for recording biopotential signals used to create propagation history maps (lower right panel). This figure was reprinted with permission from [10]

continuously rotates the catheter back and forth for approximately $3 \mathrm{~min}$. During this time, the ultrasound subsystem samples the endocardial surface at a rate of up to 115,000 surface points per minute. The location of each surface point is determined by the time it takes an acoustic wave to travel from an ultrasound transducer to the endocardial surface and back. The ultrasound point-set is subsequently transformed into a surface mesh of the atrial chamber. After minimal post processing (removal of unnecessary points, labelling of anatomical structures), the map is ready to be used as a canvas for the second part.

For this part, the basket is placed in the centre of the atrium and a quadripolar catheter is placed in the coronary sinus as electrical reference. Then, unipolar intracardiac potentials across the endocardial surface are measured by the 48 biopotential electrodes at a rate of 150,000 samples per second. This again takes approximately $2-3 \mathrm{~min}$, depending on the cycle length chosen. After data are recorded, inverse and forward algorithms are applied. For example, the QRS complex is removed to enable continuous display of activation wave fronts. The calculated data are spatially and temporally applied to the final processed surface anatomy as either a dipole density or voltage depolarisation map, or a propagation-history map. In a depolarisation map, the red region depicts the negative phase of dipole density, which, when animated, is associated with propagation of depolarisation. In the propagation-history map, red represents the leading edge of the activation wave front, with the trailing colour bands showing past locations of the wave front (Fig. 1, [9]).

\section{Results}

A total of 21 patients (age (mean \pm standard deviation [SD]) $62 \pm 8$ years, $23 \%$ female) were included. Four- 

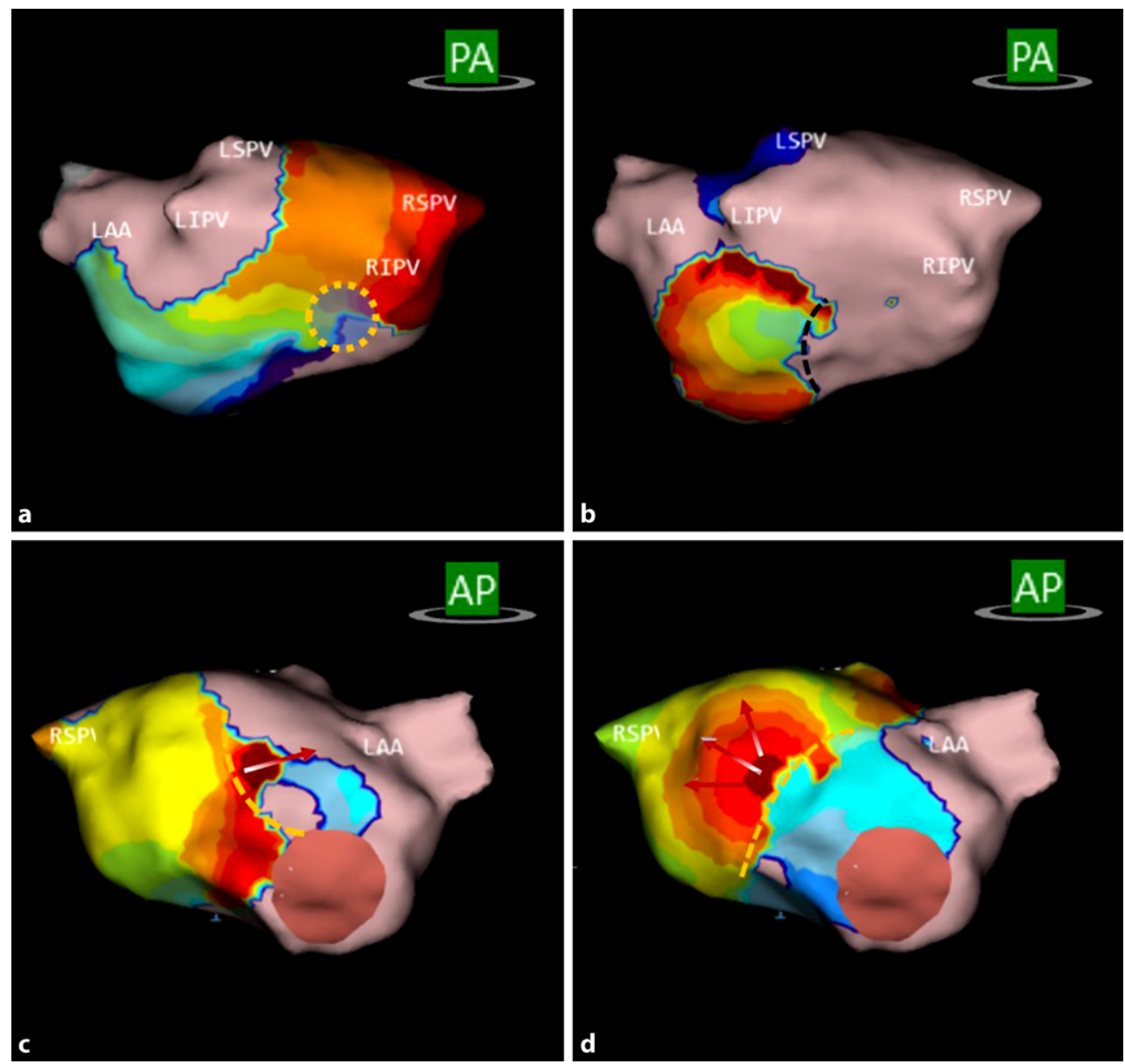

Fig. 2 Three activation patterns of interest in patient 21. All panels show propagation-history maps of the left atrium, which use bands of colour to show location and velocity of leading edge of wave front over a set period of time. Red colour indicates leading edge of wave front, with trailing colour bands showing earlier locations. Width of colour bands indicates conduction velocity of wave fronts; wider bands are indicative of fast conduction and narrow bands of slow conduction. a Localised rotational activation (spiralling around

teen patients $(67 \%)$ were treated for persistent $\mathrm{AF}$ and 7 (33\%) for atypical atrial flutter. Ablations were conducted with an irrigated ablation catheter with contact force measurement.

\section{Patients treated for persistent atrial fibrillation}

\section{Clinical characteristics}

Baseline characteristics of the 14 patients treated for persistent AF (mean \pm SD age $64 \pm 8$ years, $21 \%$ female) are shown in Tab. 1 . Eleven patients $(79 \%)$ had undergone at least one prior ablation procedure and 4 (29\%) had undergone at least two prior ablation procedures. Mean \pm SD left atrial volume index was $36 \pm 8 \mathrm{~mL} / \mathrm{m}^{2}$.

\section{The procedure}

Median (interquartile range [IQR]) procedural time was $180 \mathrm{~min}$ (156-218), and median (IQR) radiofrequency ablation time was $44 \mathrm{~min}$ (21-79) (see Tab. S1 a small, confined zone, indicated by dotted yellow circle) in the posterior wall of left atrium $(L A)$. $\mathbf{b}$ Focal activation in posterior wall of LA. c, $\mathbf{d}$ Localised irregular activation (entry/exit through confined zone, indicated by red arrows) in anterior wall of $L A$. PA posteroanterior, $A P$ anteroposterior, $L A A$ left atrial appendage, $L I P V$ left inferior pulmonary vein, $L S P V$ left superior pulmonary vein, RIPV right inferior pulmonary vein, $R S P V$ right superior pulmonary vein

in the Electronic Supplementary Material). All patients had AF at the start of the procedure. Following PVI or re-PVI, atrial activation patterns of interest (APIs) were targeted. These included focal, localised rotational activation (spiralling around a small, confined zone $\geq 270^{\circ}$ ) and localised irregular activation (entry/exit through and pivoting around a confined zone). These APIs were ablated separately at each remapped stage and, where possible, connected to near PVI circumferential lesions and/or anatomical barriers. Fig. 2 shows examples of the three mechanisms in patient 21. The number of mechanisms in all 14 patients by location is shown in Fig. S1 (see Electronic Supplementary Material). If conversion to sinus rhythm did not occur after ablation of all APIs, electrocardioversion was performed. 
Table 1 Baseline characteristics of 14 patients treated for persistent atrial fibrillation and 7 patients treated for atypical atrial flutter

\begin{tabular}{|c|c|c|c|c|c|}
\hline $\begin{array}{l}\text { Patient } \\
\text { number }\end{array}$ & $\begin{array}{l}\text { Diag- } \\
\text { nosis }\end{array}$ & $\begin{array}{l}\text { Age, } \\
\text { years }\end{array}$ & Sex & Clinical history & $\begin{array}{l}\mathrm{LAVI} \\
\mathrm{mL} / \mathrm{m}^{2}\end{array}$ \\
\hline 1 & $\mathrm{AF}$ & 59 & M & PVI, re-PVI, VCS isolation & NA \\
\hline 2 & $\mathrm{AF}$ & 68 & $\mathrm{~F}$ & PVI & 33 \\
\hline 3 & AFL & 69 & M & $\begin{array}{l}\text { Mini-Maze (PVI + box + trigonum } \\
\text { line + bicaval line) }\end{array}$ & 45 \\
\hline 4 & $\mathrm{AF}$ & 47 & M & PVI & 49 \\
\hline 5 & $\mathrm{AF}$ & 70 & $\mathrm{~F}$ & PVI & 32 \\
\hline 6 & $\mathrm{AF}$ & 54 & M & None & 25 \\
\hline 7 & $\mathrm{AF}$ & 52 & M & None & 43 \\
\hline 8 & $\mathrm{AF}$ & 61 & M & None & 27 \\
\hline 9 & AFL & 68 & M & PVI & NA \\
\hline 10 & AF & 57 & M & $\begin{array}{l}\text { PVI, mini-Maze (PVI + box + gan- } \\
\text { glion plexus ablation) }\end{array}$ & 44 \\
\hline 11 & AF & 77 & M & PVI & NA \\
\hline 12 & $\mathrm{AF}$ & 66 & $\mathrm{~F}$ & $\begin{array}{l}\mathrm{PVI} \text {, mini-Maze (PVI + box + } \\
\text { trigonum line + bicaval line + } \\
\text { RA line) }\end{array}$ & 33 \\
\hline 13 & AFL & 69 & M & $\begin{array}{l}\mathrm{PVI} \text {, re-PVI, mini-Maze (PVI + box } \\
+ \text { trigonum line) }\end{array}$ & 67 \\
\hline 14 & AFL & 68 & M & PVI, CTI ablation, mini-Maze & NA \\
\hline 15 & AF & 65 & M & $\begin{array}{l}\text { Mini-Maze (PVI + box + trigonum } \\
\text { line + bicaval line + RA line) }\end{array}$ & 43 \\
\hline 16 & AFL & 78 & $\mathrm{~F}$ & $\begin{array}{l}\text { PVI, re-PVI, CTI ablation, mini- } \\
\text { Maze }\end{array}$ & 20 \\
\hline 17 & $\mathrm{AF}$ & 68 & M & PVI & 29 \\
\hline 18 & $\mathrm{AF}$ & 75 & M & PVI & NA \\
\hline 19 & AFL & 61 & $\mathrm{~F}$ & $\begin{array}{l}\mathrm{PVI} \text {, mini-Maze (PVI + box + } \\
\text { trigonum line + bicaval line) }\end{array}$ & 30 \\
\hline 20 & AFL & 66 & M & $\begin{array}{l}\text { PVI, re-PVI, CTI ablation, re-re-PVI, } \\
\text { mini-Maze (PVI + box) }\end{array}$ & 32 \\
\hline 21 & AF & 71 & M & $\begin{array}{l}\text { PVI, re-PVI, CryoMaze (PVI + box + } \\
\text { trigonum line) }\end{array}$ & 46 \\
\hline
\end{tabular}

$A F$ atrial fibrillation, $A F L$ atrial flutter, $P V /$ pulmonary vein isolation, VCS vena cava superior, $L A V /$ left atrial volume index, $R A$ right atrial, $C T /$ cavotricuspid isthmus

\section{Procedural success}

Two patients (patients 10 and 12) converted to sinus rhythm during ablation. At the end of the procedure, a total of 11 patients $(79 \%)$ were in sinus rhythm (see Tab. S1 in the Electronic Supplementary Material). In the remaining 3 patients, AF persisted despite electrocardioversion. In one of these patients, the right inferior pulmonary vein had a very low take-off, which made it impossible to isolate. In the other 2 patients APIs kept on returning despite extensive ablation.

At the 3-month follow-up point, 5 patients (36\%) were in sinus rhythm (Tab. 2). Of the patients with no prior ablation procedure or only one prior PVI, 3 (33\%) were in sinus rhythm after 3 months. Of the patients with multiple prior ablation procedures, $2(40 \%)$ were in sinus rhythm after 3 months. Five patients $(36 \%)$ underwent a redo procedure around 6 months of follow-up. At the 12-month follow-up point, 4 patients (without redo procedure) (29\%) remained in sinus rhythm.
Table 2 Outcomes of 14 patients treated for persistent atrial fibrillation

\begin{tabular}{|c|c|c|c|c|}
\hline $\begin{array}{l}\text { Patient } \\
\text { number }\end{array}$ & 3 months & 6 months & 9 months & 12 months \\
\hline 1 & $\mathrm{AF}(\mathrm{ECG})$ & $\mathrm{AF}(\mathrm{ECG})$ & $\mathrm{AF}(\mathrm{ECG})$ & $\mathrm{AF}(\mathrm{ECG})$ \\
\hline 2 & $\mathrm{SR}(\mathrm{ECG})^{\mathrm{a}}$ & NA & SR (Holter) & SR (ECG) \\
\hline 4 & AFL (ECG) & Redo ablation & - & - \\
\hline 5 & $\mathrm{AF}(\mathrm{ECG})$ & Mini-Maze & - & - \\
\hline 6 & $\mathrm{SR}(\mathrm{ECG})^{\mathrm{a}}$ & SR (Holter) ${ }^{\mathrm{a}}$ & SR (ECG) & SR (ECG) \\
\hline 7 & AF (ECG) & $\mathrm{SR}(\mathrm{ECG})^{\mathrm{a}}$ & $\begin{array}{l}\text { Paroxysmal AF } \\
\text { (Holter) }\end{array}$ & SR (Holter) ${ }^{\mathrm{a}}$ \\
\hline 8 & AFL (ECG) & Redo ablation & - & - \\
\hline 10 & $\begin{array}{l}\text { SR } \\
\text { (pacemaker) }^{\mathrm{a}}\end{array}$ & NA & SR (pacemaker) & SR (pacemaker) \\
\hline 11 & $\mathrm{SR}(\mathrm{ECG})^{\mathrm{a}}$ & SR (ECG) & SR (ECG) & $\mathrm{AF}$ (ECG) \\
\hline 12 & SR (ECG) & NA & NA & SR (ECG) \\
\hline 15 & $\mathrm{AF}(\mathrm{ECG})$ & Redo ablation & - & - \\
\hline 17 & AF (ECG) & $\begin{array}{l}\text { Persistent AF } \\
\text { (pacemaker) }\end{array}$ & $\begin{array}{l}\text { Persistent AF } \\
\text { (pacemaker) }\end{array}$ & $\begin{array}{l}\text { Persistent AF } \\
\text { (pacemaker) }\end{array}$ \\
\hline 18 & AF (ECG) & Mini-Maze & - & - \\
\hline 21 & $\mathrm{AF}(\mathrm{ECG})$ & AFL (ECG) & NA & NA \\
\hline
\end{tabular}

$A F$ atrial fibrillation, $E C G$ electrocardiography, $S R$ sinus rhythm, NA not available, $A F L$ atrial flutter

a Patient was on antiarrhythmic drugs

Table 3 Outcomes of 7 patients treated for atypical atrial flutter

\begin{tabular}{|c|c|c|c|c|}
\hline $\begin{array}{l}\text { Patient } \\
\text { number }\end{array}$ & 3 months & 6 months & 9 months & 12 months \\
\hline 3 & SR (ECG) & NA & SR (ECG) & SR \\
\hline 9 & $\mathrm{SR}(\mathrm{ECG})^{\mathrm{a}}$ & SR (ECG) & SR (Holter) & SR (ECG) \\
\hline 13 & SR (ICD) & $\mathrm{SR}(\mathrm{ICD})$ & SR (ICD) & SR (ICD) \\
\hline 14 & AFL (ECG) & NA & NA & $\mathrm{AF}(\mathrm{ECG})$ \\
\hline 16 & $\begin{array}{l}\text { Persistent AF } \\
\text { (pacemaker) }\end{array}$ & NA & NA & $\begin{array}{l}\text { Persistent AF } \\
\text { (pacemaker) }\end{array}$ \\
\hline 19 & Redo ablation & - & - & - \\
\hline 20 & $\begin{array}{l}\text { SR (pace- } \\
\text { maker) }\end{array}$ & SR (pacemaker) & SR (pacemaker) & SR (pacemaker) \\
\hline
\end{tabular}

\section{Patients treated for atypical flutter}

\section{Clinical characteristics}

Baseline characteristics of the 7 patients treated for atypical flutter (mean \pm SD age $68 \pm 5$ years, $29 \%$ female) are shown in Tab. 1. All patients had undergone at least one prior ablation procedure and $5(71 \%)$ had undergone at least two procedures. Mean \pm SD left atrial volume index was $38 \pm 18 \mathrm{~mL} / \mathrm{m}^{2}$.

\section{The procedure}

Median (IQR) procedural time was 205 min (190-215), and median (IQR) radiofrequency ablation time was 11 min (10-16) (see Tab. S1 in the Electronic Supplementary Material). Five patients had atrial flutter at the start of the procedure. In the remaining 


\section{Advertisement placed here.}

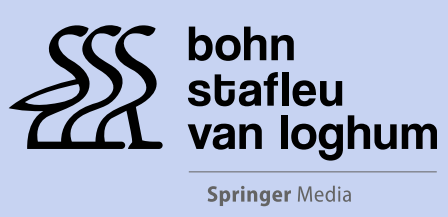

Houten 2021 


\section{Advertisement placed here.}

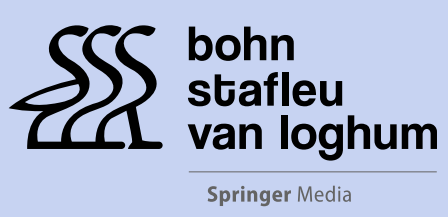

Houten 2021 

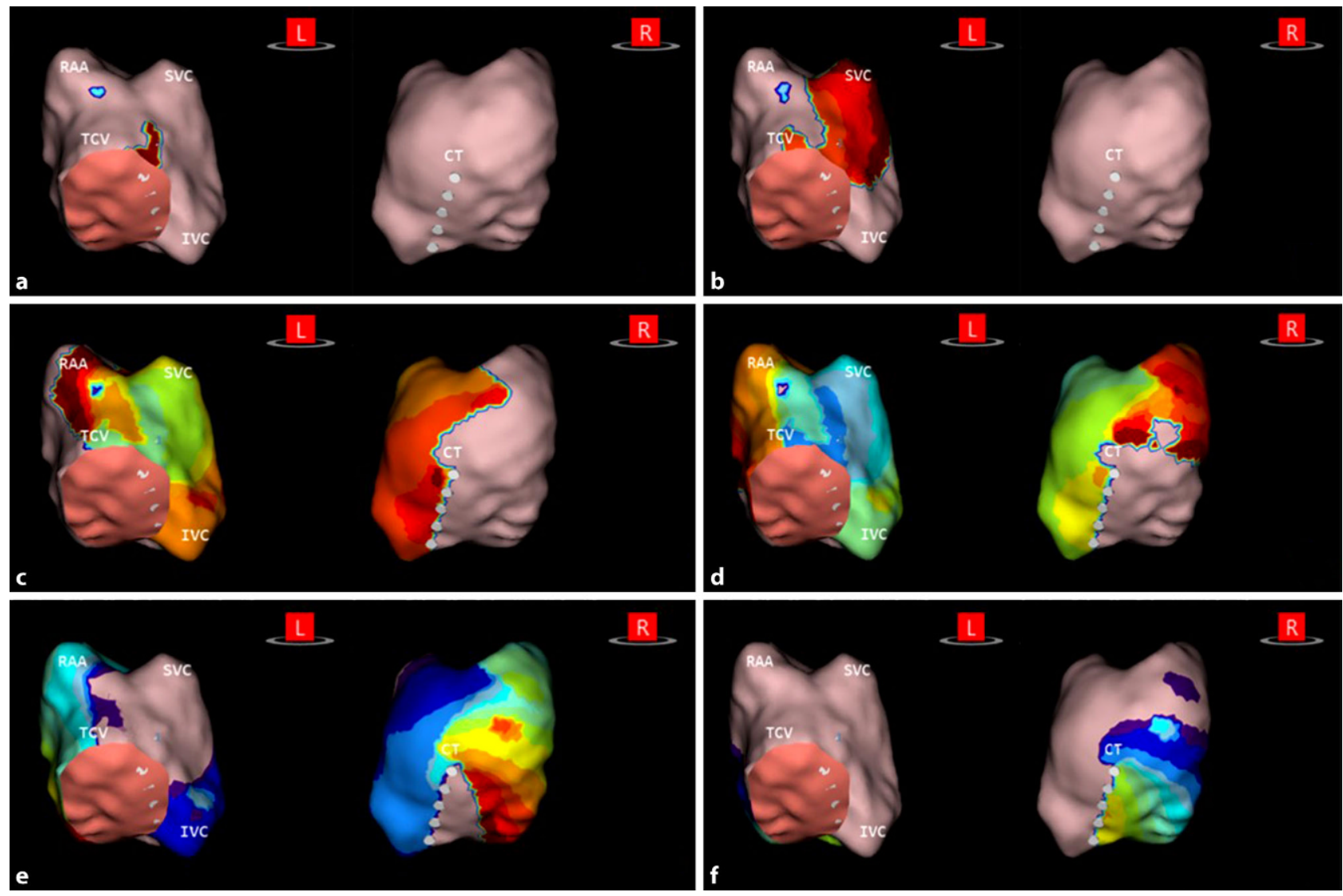

Fig. 3 Example of right-sided atypical flutter in patient 16. Propagation-history map of right atrium, which uses bands of colour to show location and velocity of leading edge of wave front over a set period of time. Red colour indicates leading edge of wave front, with trailing colour bands showing earlier locations. Width of colour bands indicates conduction velocity of wave fronts; wider bands are indicative of fast conduction and narrow bands of slow conduction. a Focal activation at tri-

2 patients, atrial flutter was provoked by incremental atrial pacing. Median (IQR) cycle length was $251 \mathrm{~ms}$ (200-260). In 3 patients, ablation was performed in the left atrium, in 3 patients in the right atrium, and in 1 patient in both. Fig. 3 shows an example of a rightsided atypical flutter in patient 16 (for the complete video, see Electronic Supplementary Material).

\section{Procedural success}

Acute success, defined as sinus rhythm without the ability to provoke the clinical arrhythmia, was achieved in 6 patients (86\%) (see Tab. S1 in the Electronic Supplementary Material). At the 3-month follow-up point, 4 patients (57\%) were in sinus rhythm, and these patients remained in sinus rhythm during 12 months of follow-up (Tab. 3). One patient (14\%) had a recurrence of the clinical arrhythmia and later developed AF. The remaining 2 patients also developed $\mathrm{AF}$, for which one of them underwent a redo ablation.

cuspid annulus. b Propagation of wavefront towards superior vena cava (SVC). c Separation into two wavefronts towards inferior septum and lateral wall, respectively. d Propagation of two wavefronts down septum and lateral wall. e, f Collision of two wavefronts at cavotricuspid isthmus. $L$ left, $R$ right, $R A A$ right atrial appendage, TCV tricuspid valve, IVC inferior vena cava, $C T$ crista terminalis

\section{Complications}

All complications are listed in Table S1 (see Electronic Supplementary Material). There were 2 patients $(10 \%)$ with major complications attributable to radiofrequency ablation. Both were treated for an atypical flutter; one patient developed hemiparesis for which intra-arterial thrombolysis was given with subsequent good clinical outcome. The same patient and one other patient developed complete atrioventricular block, for which a permanent pacemaker was implanted. No major complications attributable to the mapping system occurred.

\section{Discussion}

Persistent AF is a complex arrhythmia, with drivers and maintenance mechanisms outside the pulmonary veins [3-5]. Conventional point-by-point mapping is inadequate to understand these mechanisms, and empiric ablation has been disappointing [6]. Global mapping of $\mathrm{AF}$ could overcome the limitations of 
point-by-point mapping and facilitate a patient-tailored approach.

The first noncontact global mapping system was the EnSite Array (Abbott/St. Jude Medical Inc., St. Paul, MN, USA), which comprises 64 unipolar electrodes [11]. It displays calculated endocardial unipolar electrograms on a surface that is physically traced by a conventional catheter. The main limitation of this technology is that the precision of the calculated electrograms depends on the distance from the endocardial surface. Moreover, it has only been validated for distances $<40 \mathrm{~mm}$ [12].

The FIRMap Catheter (with the use of the Topera Rhythm View 3D Mapping System, Abbott/Topera Medical, Palo Alto, CA, USA) was the first to identify spiral rotors and focal drivers of $\mathrm{AF}$ and facilitate patient-specific ablation in humans. The CONFIRM study showed an acute success rate of $86 \%$ and freedom from $\mathrm{AF}$ in $83 \%$ of the patients after a median follow-up period of 273 days [13]. However, more recent studies have failed to achieve acute and longterm outcomes similar to those seen in the CONFIRM study [14-18]. A possible explanation for the disappointing results from these studies is that the FIRMap Catheter is a contact basket, which is meant to be a 'one size fits all'. Several studies have reported poor contact by the basket catheter electrodes and clustering of splines [15, 18].

The AcQMap High Resolution Imaging and Mapping System is the latest addition to the arsenal of global AF mapping systems. This system provides 3D maps of electrical activation across an ultrasoundacquired atrial surface. The acquisition of the ultrasound surface map takes 2-3 min, after which consecutive activation maps can be generated on a beat-tobeat basis. Another novel aspect of the system is that it can generate dipole density maps in addition to the conventional voltage maps.

A dipole consists of two oppositely charged particles separated by a very small distance. Each time a cell is stimulated, ions move across the cellular membrane, causing a small dipolar imbalance in the adjoining extracellular medium. The combined and consecutive activation of multiple cells forms a macroscopic double layer of dipoles that directly represents the wave front and, in turn, generates the cardiac potential field, measured in volts. The differences between voltage and dipole density lie in both the averaging effect of 'spatial summation' and in the volume of space occupied by each. Spatial averaging causes the distribution of voltage to extend far beyond the compact, physical boundary of the charges. It also smooths out some of the localised details of the geometric shape of the wave front. This explains why it is possible to measure the heart's electrocardiographic signals with an ECG, although with significantly less spatial detail than that of intracardiac electrograms [19].
The AcQMap System is able to generate dipole density maps because it can simultaneously measure the endocardial potential field and the anatomic surface. This enables spatially localised and temporally animated derivation of the dipolar charge sources on the endocardial surface, which can be displayed as waves of activation across a reconstructed 3D surface through time.

\section{Recurrence rate}

In our experience, the AcQMap System is user-friendly and safe. Atypical flutter circuits and APIs were easily identified, and the acute success rate was high. Of the patients treated for atypical flutter, only one patient had a recurrence of the clinical arrhythmia during follow-up. The outcome of the patients treated for persistent AF was less optimal. After 12 months, only $29 \%$ of the patients were in sinus rhythm. One possible explanation for this high recurrence rate is that the studied population had already been found to be therapy-resistant (79\% had undergone at least one prior ablation procedure). Moreover, the recently published UNCOVER trial, which included 127 patients with persistent AF who underwent de novo catheter ablation using the AcQMap System, showed a single-procedure freedom from AF rate of $73 \%$ at 12 months [20].

Another possible, but less hopeful, explanation for the high recurrence rate in our cohort is that persistent $\mathrm{AF}$ is not the resultant of single or small numbers of stable localised APIs, but that their location varies over time as the substrate may keep deteriorating. Observations by Haissaguerre et al. point in this direction [21, 22]. They used a body surface mapping system with 252 external electrodes in combination with computed tomography to record biatrial unipolar electrograms and create simultaneous biatrial 3D activation maps. These maps showed incessantly changing beat-to-beat wavefronts and varying spatiotemporal behaviour of driver activities. The rotors they found had a median duration of 2.6 rotations, as opposed to the minutes to hours in the CONFIRM study. The authors also found that the number of targeted driver regions increased with the duration of persistent AF ( 2 in patients presenting in sinus rhythm, 3 in AF lasting 1 to 3 months, 4 in AF lasting 4 to 6 months, and 6 in AF lasting longer), while the termination rate sharply declined after 6 months. The observation that AF begets AF is not new, nor is the observation that with prolonged duration of $\mathrm{AF}$, the substrate becomes more complex [23, 24]. Therefore, together with a good method for mapping and targeting drivers of persistent AF, early timing may also be of the essence.

\section{Study limitations}

This study has several important limitations. Data collection was limited to variables that were routinely 
collected and was incomplete in some patients. The studied population was small, and no comparisons with other ablation strategies or mapping systems were made. Therefore, conclusions with regard to safety and efficacy cannot be drawn based on this study alone, and our report should be interpreted as a feasibility pilot study.

\section{Conclusion}

The AcQMap System is able to provide fast, high-resolution activation maps of persistent AF and atypical atrial flutter. Despite a high acute success rate, the recurrence rate of persistent $\mathrm{AF}$ in this selected, therapy-resistant population was relatively high. This may be due to the limited experience in the optimal use of this novel system and the selection of a very difficult patient population that had failed all prior treatment strategies.

Conflict of interest M.Liebregts, M.C.E.F.Wijffels, M.N. Klaver, V.F. van Dijk, J.C. Balt and L.V.A. Boersma declare that they have no competing interests.

Open Access This article is licensed under a Creative Commons Attribution 4.0 International License, which permits use, sharing, adaptation, distribution and reproduction in any medium or format, as long as you give appropriate credit to the original author(s) and the source, provide a link to the Creative Commons licence, and indicate if changes were made. The images or other third party material in this article are included in the article's Creative Commons licence, unless indicated otherwise in a credit line to the material. If material is not included in the article's Creative Commons licence and your intended use is not permitted by statutory regulation or exceeds the permitted use, you will need to obtain permission directly from the copyright holder. To view a copy of this licence, visit http://creativecommons.org/licenses/by/4.0/.

\section{References}

1. Cosedis Nielsen J, Johannessen A, Raatikainen P, Hindricks G, Walfridsson H, Kongstad O, et al. Radiofrequent ablation as initial therapy in paroxysmal atrial fibrillation. NEngl J Med. 2012;367:1587-95.

2. Pérez FJ, Schubert CM, Parvez B, Pathak V, Ellenbogen KA, Wood MA. Long-term outcomes after catheter ablation of cavo-tricuspid isthmus dependent atrial flutter: a metaanalysis. Circ Arrhythm Electrophysiol. 2009;2:393-401.

3. GrayRA, PertsovAM, Jalife J. Spatial and temporal organization during cardiac fibrillation. Nature. 1998;392:75-8.

4. Botteron GW, Smith JM. Quantitative assessment of the spatial organization of atrial fibrillation in the intact human heart. Circulation. 1996;93:513-8.

5. Sanders P, Berenfeld O, Hocini M, et al. Spectral analysis identifies sites of high-frequency activity maintaining atrial fibrillation in humans. Circulation. 2005;112:789-97.

6. Oral H, Pappone C, Chugh A, et al. Circumferential pulmonary-vein ablation for chronic atrial fibrillation. NEngl J Med. 2006;354:934-41.

7. Wynn GJ, Das M, Bonnett LJ, Panikker S, Wong T, Gupta D. Efficacy of catheter ablation for persistent atrial fibrillation: a systematic review and meta-analysis of evidence from randomized and nonrandomized controlled trials. Circ ArrhythmElectrophysiol. 2014;7:841-52.
8. Grace A, Willems S, Meyer C, et al. High-resolution noncontact charge-density mapping of endocardial activation. JCI Insight. 2019;4:e126422. https://doi.org/10.1172/jci. insight.126422.

9. Grace A. Modifying PVI lines to incorporate non-PV targets identified by pre-ablation mapping with the acQmap system: update on the UNCOVER-AF trial. Ep Lab Dig. 2017;17:40-3. https://www.eplabdigest. com/articles/Modifying-PVI-Lines-Incorporate-NonPV-Targets-Identified-Pre-Ablation-Mapping-AcQMap.

10. Grace A. Modifying PVI lines to incorporate non-PV targets identified by pre-ablation mapping with the AcQMap system: update on the UNCOVER-AF trial. EP Lab Digest. 2017;17:40-3.

11. Hindricks G, Kottkamp H. Simultaneous noncontact mapping of left atrium in patients with paroxysmal atrial fibrillation. Circulation. 2001;104:297-303.

12. Earley MJ, Abrams DJ, Sporton SC, Schilling RJ. Validation of the noncontact mapping system in the left atrium during permanent atrial fibrillation and sinus rhythm. J Am Coll Cardiol. 2006;48:485-91.

13. Narayan SM, Krummen DE, Shivkumar K, Clopton P, Rappel WJ, Miller JM. Treatment of atrial fibrillation by the ablation of localized sources: CONFIRM (Conventional Ablation for Atrial Fibrillation With or Without Focal Impulse and Rotor modulation) trial. J Am Coll Cardiol. 2012;60:628-36.

14. Tilz RR, Lin T, Rillig A, et al. Nine month outcomes following focal impulse and rotor modulation for the treatment of atrial fibrillation using the novel 64-pole basket catheter. Europace. 2015;17:iii16.

15. Kuklik P, Van Hunnik A, Zeemering S, et al. Technical challenges of rotor identification during atrial fibrillation using phase singularity detection. Europace. 2015;17:ii20.

16. Schade A, Halbfass P, Mueller P, et al. FIRM only ablation in patients with persistent atrial fibrillation: acute and medium-term results. Europace. 2015;17:iii68.

17. Gianni C, Mohanty S, Di Biase L, et al. Acute and shortterm outcomes in persistent and long-standing persistent patients undergoing rotors only ablation. Heart Rhythm. 2016;13:830835.

18. Buch E, Share M, Tung R, et al. Long-term clinical outcomes of focal impulse and rotor modulation for treatment of atrial fibrillation: a multicenter experience. Heart Rhythm. 2016;13:636-41.

19. Shi R, Norman M, Wong T. Individualized ablation strategy guided by live simultaneous global mapping to treat persistent atrial fibrillation. Future Cardiol. 2018;14:237-49.

20. WillemsS, VermaA, Betts TR, etal. Targetingnonpulmonary vein sources in persistent atrial fibrillation identified by noncontact charge density mapping. Circ Arrhythm Electrophysiol. 2019;12:e7233.

21. Haissaguerre $\mathrm{M}$, Shah $\mathrm{AJ}$, Cochet $\mathrm{H}$, et al. Intermittent drivers anchoring to structural heterogeneities as a major pathophysiological mechanism of human persistent atrial fibrillation. J Physiol. 2016;594:2387-98.

22. Haissaguerre M, Hocini M, Denis A, et al. Driver domains in persistent atrial fibrillation. Circulation. 2014;130:530-8.

23. Wijffels MC, Kirchhof CJ, Dorland R, Allessie MA. Atrial fibrillation begets atrial fibrillation. Astudy in awake chronically instrumented goats. Circulation. 1995;92:1954-68.

24. Verheule S, Tuyls E, van Hunnik A, Kuiper M, Schotten U, Allessie M. Fibrillatory conduction in the atrial free walls of goats in persistent and permanent atrial fibrillation. Circ Arrhythm Electrophysiol. 2010;3:590-9. 